\title{
Handoff Prioritize Call Admission Control Policy for Integrated Macrocell-Femtocell Network
}

\author{
Kranti Bhoite \\ PhD Research Scholar \\ Bharati Vidyapeeth Deemed University \\ COE, Pune- 411043, India
}

\author{
Sachin Gengaje, PhD \\ Dept. of Electronics Engineering. \\ Bharati Vidyapeeth Deemed University \\ COE, Pune- 411043 , India
}

\begin{abstract}
Latest wireless network technologies facing network capacity issue \& not able to achieve its goal of delivering higher data rate due to poor indoor coverage issue. Femtocells are less expensive alternative for achieving high data rate and better indoor coverage. Femtocells are home base stations placed near to subscriber which supplements existing macrocell network. It normally has three different access modes called Open access, closed access \& hybrid access mode. Call Admission control (CAC) in integrated macrocell-femtocell need special attention due to these access modes \& used to ensure better quality of service for mobile subscribers. In this article, we will study registered users handoff call prioritization CAC policy. The proposed policy considers hybrid access mode for femtocell network and gives high priority to femtocell register user. It uses guard or reserved channel mechanism \& buffer concept for call admission control. Comparison of handoff prioritized scheme (HPS) and non-prioritized schemes (NPS) \& benefits of handover prioritized scheme over non-prioritized schemes are highlighted in this article.
\end{abstract}

\section{General Terms}

Femtocell Network, Wireless Communication, Integrated Macrocell-Femtocell network

\section{Keywords}

Call Admission Control, Handover Management, Femtocell network, Femtocell Access Modes.

\section{INTRODUCTION}

With latest wireless technologies, new demands for quality of service have been created. As per Global Mobile Data Traffic Forecast by Cisco which talks about major global mobile data traffic projections and growth trends, there was $63 \%$ growth in global mobile data traffic in 2016 [1]. To address this high capacity demand macrocell network is not enough. Femtocell network supplement this macrocell network to fulfill high capacity demand \& to achieve better indoor coverage. Femtocells are low power home base stations (HBS) which are placed indoor [2]. As femtocells are placed closed to end users, path loss is less. Femtocells combine the internet access technologies and mobile technologies and routes call through internet access technology when in femtocell coverage area. Femtocells work in three different access modes [3]

- Open Access Mode: It provide services to subscribers of any PLMN. It's simple \& no additional configuration is needed. The only limiting factor is capacity of the femtocell and the capacity of the backhaul connection.

- Closed Access Mode: It provide service to only registered users of femtocell that belong to its associated close subscriber group (CSG). The CSG manager shall be able to add, remove and view CSG membership. Inefficient use of spectrum reserved for CSG in case there are very less CSG Members.

- Hybrid Access Mode: In this mode, services are provided to its registered users that belong to its associated close subscriber group (CSG), and other non-registered users of any PLMN which are not belonging to its associated CSG. When the load levels in femtocell rise the users belonging to the CSG get better service.

Call Admission control in femtocell network need some modifications to conventional call admission approaches due to three different access modes. In integrated macrocell femtocell when a user needs new connection \& raise request for it, CAC first check the access mode of femtocell and whether user is allowed to access the femtocell network. Later it calculate the number of resources required by

(i) Existing users in the network \& its resource requirement

(ii) Users waiting for services

Users connection request is fulfilled only when the sum of the existing users \& waiting users required capacity is not larger than the total capacity, otherwise, the request will be rejected [4]. In this article, handoff priorities call admission control is proposed for hybrid access mode. For users point of view it's more annoying to get disconnected in between than getting congestion issue at starting hence this approach gives higher priority to handoff calls \& also as hybrid access mode is considered, higher priority is given to registered users of femtocell. The rest of this paper is planned as follows: Markov chain model is studied in Section 2. It gives a brief introduction to Markov Model. It can evaluate blocking probabilities of hand-off call \& new call separately. Section 3 describes the proposed handoff prioritization Call Admission Control policy for hybrid access mode of femtocell network in details, while section 4 shows the system model as well as simulation model when all components are put together with a modeling scenario. Finally this paper concludes with benefits of the proposed CAC policy, its role in QoS provisioning in terms of the signal quality, new call blocking and handover call blocking probability.

\section{MARKOV MODEL}

Let us consider femtocell network with hybrid access mode randomly deployed on pre-planned macrocell network. For this work, we will prepare one multidimensional Markov chain model which will help us to compute handoff call blocking probability new-call blocking probability for registered \& non-registered users. Instead of handling new/hand-off calls and registered/non-registered users request similarly, we will add the idea of few reserved channels for handoff calls \& registered user. In integrated macrocellfemtocell network within its coverage area, macro cellular base station (BS) and femtocell access point serves various subscribers, multimedia service and number of mobile 
terminals. Cellular network is already exhausted with high capacity demand \& less resources. Also due to handover feature in wireless cellular network when a user moves from one cell to another, if certain criteria's are met then the control or communication of mobile is transferred to another cell. For this next cell needs to allocate sufficient resources for taking the responsibility of established connection(s) [5]. Let's assume that potential customers are uniformly distributed over the integrated macrocell and femtocell coverage area, to model hand-offs.

Let's calculate Traffic per user and total traffic to build markov chain model.

- Traffic per user $=$ User call rate * Average call duration

- $\quad$ Total Traffic $=$ Total number of users $*$ Traffic per user

Maximum one earlang traffic can be handled by single communication channel. Hence N Earlang of traffic can be supported by $\mathrm{N}$ channels.

Let's assume, New and handoff call arrival rate is modeled by the Poisson distribution and Call arrival rate is ' $K$ '. Hence, ' $X$ ' call arrival in a time duration ' $\Delta \mathrm{t}$ ' is given by Discrete Distribution defined or Poisson Distribution $\mathrm{P}(\mathrm{x})$ for $\mathrm{x}=$ $0,1,2 \ldots \ldots \infty$

$P(x)=\frac{(\kappa \Delta t)^{x}(e)^{-K \Delta t}}{x !}$

The number of channel occupied at a given point of time is the state of wireless system. If the max number of channel in the wireless system is $C$ then we can have multiple states such as $\mathrm{S} 0, \mathrm{~S} 1 \ldots \mathrm{S}_{\mathrm{C}}$ in markov chain model [6]. Here,

$\mathrm{S} 0=$ State when no channels are occupied in system

$\mathrm{Sc}=$ State when all channels are occupied in system.

$\mathrm{Sk}=$ State when $\mathrm{x}$ channels are occupied in system

Let's see markov chain model \& its states in following Fig. 1

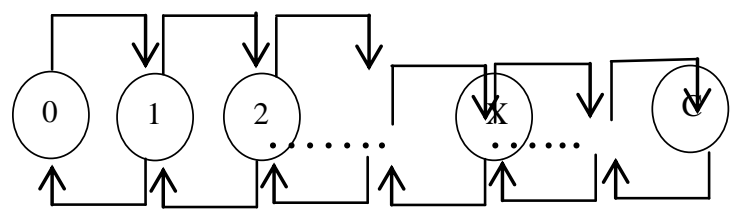

Fig 1. State Space Diagram of Markov Chain

The transition of states from 0 to $\mathrm{C}$ (max channels) when call request arise is shown in state space diagram. When all channels are vacant then it is shown by state zero $S_{0} \&$ that state transition is done one from zero when new call arises. At the time of one call departs from state one then it transition state zero from state one \& so on [6]. Once all total C channels are occupied then no new calls can be addressed, this is nothing but the Blocking Probability $\mathrm{P}(\mathrm{C})$.

The probabilities of all channels are occupied i.e. Blocking Probability is given by

$$
P(C)=\frac{(K \Delta t)^{C}(e)^{-K \Delta t}}{C !}
$$

Similarly, by using steady state analysis \& Poisson distribution we can calculate new call arrival probability. Let's consider an infinitesimally small time interval $\Delta$ t. With this small time interval let's calculate Probability of one call arrival based on markov chain model.
$\mathrm{P}($ One call arrival $)=\frac{(\kappa \Delta \mathrm{t})^{1}(\mathrm{e})^{-\Lambda \Delta \mathrm{t}}}{1 !}$

$$
=(\Lambda \Delta t)^{1}(e)^{-\Lambda \Delta t}
$$

Since, $-\lambda \Delta \mathrm{t}$ is very small, we can say that $(\mathrm{e})^{-\kappa \Delta \mathrm{t}} \approx 1$

$\mathrm{P}($ One call arrival $) \approx \lambda(\Delta \mathrm{t})$

In short Probability one call arrives in time $\Delta \mathrm{t}$ is $\lambda \Delta \mathrm{t}$.

State Transition Probability Formulation of Markov chain is shown as below,

$P(j) \begin{cases}\frac{K(\Delta t)}{} & \text { When one channel is occupied } \\ \frac{(K \Delta t)^{x}(e)^{-K \Delta t}}{x !} & \text { When } x \text { channels are occupied } \\ \frac{(K \Delta t)^{N}(e)^{-K \Delta t}}{N !} & \text { When all channels are occupied }\end{cases}$

Where,

$\mathrm{j}=$ State of Markov Chain Model

$\lambda=$ Rate of call arrival in integrated macro-femto network

$\mathrm{x}=$ number of states

$\mathrm{t}=$ time duration

$\Delta \mathrm{t}=$ time duration for one call

\section{PROPOSED HANDOFF PRIORITIZE} CALL ADMISSION CONTROL

Femtocells are installed in predominant macrocell coverage area $\&$ have very small coverage area as compare to macrocell coverage area. Due to small coverage area numbers of handovers are more \& sometime unnecessary. User can face ping pong $\mathrm{HO}$ issue because of integrated macrocell-femtocell network scenario. For this paper, handoff prioritization call admission control Scheme for integrated macrocell-femtocell network is considered. It is combination of early blocking and queuing approach. Two different CAC concept called Guard channel policy (Reservation type CAC) [7] \& buffer (Queuing type CAC) [8] are used in proposed algorithm for congestion control and better handover experience for subscriber. Twothreshold guard channel policy and queue mechanism used to compute call \& handoff blocking performance [7]. We have chosen Guard channel policy for this work due to its simplicity \& it helps us to calculate CAC policy parameters in a systematic way according to services provider specific QoS requirements.

In this section, we first introduce handoff prioritization by early blocking policy. Multi-threshold guard channel policy for handoff prioritization by early blocking is considered here and then its blocking performance is computed. For new \& handoff call arrival we have used four dimensional markov chain model. With four dimensional markov models, we can prioritize three different types of calls or services. Here in this approach, handoff calls of registered user of femtocell gets first or highest priority then next priority to new calls of registered user \& last priority is given to unregistered users.

In this approach, we consider integrated Macrocell \& femtocell network with hybrid access mode. Femtocells are deployed randomly over properly planned macrocell network. In this integrated network, mobile equipment will generate measurement Report. Based on measurement report \& 
comparing it with network threshold decided by service provider, we will decide whether handover is required or not. If handover is required for user, active set of neighbor or neighbor list for taking handover is created. Now we will apply actual call admission control approach on first best candidate for handover. In this CAC approach, we have combined two threshold guard channel policy \& queuing policy to give highest priority to handoff calls \& registered users. This approach is also useful for effective resource utilization and for reducing handoff blocking probability of closed access subscriber of femtocell network. Proposed algo not only divides total channel capacity into 3 subset but also one buffer is added to hold handoff calls. The concept of adding buffer does not allow handoff calls to reject or drop. 3 subsets are Shared channel, reserved channels and dedicated reserved channel \& finally new buffer channels are added when total channel capacity is utilized. Ordinary Channels are shared by registered as well non-registered users \& for new/handoff call access. Reserved channels reject access to non-registered user \& shared by femtocell registered users for new \& handoff calls. Dedicated reserved channels only gives access to registered handoff calls. We don't want users call to drop even if channel capacity is full so we have added buffer concept. Once max numbers of channels are full then handoff call kept in buffer till any channel gets free \& avoid handoff call drop. Fig. 2 shows flowchart of the Proposed CAC policy for integrated macrocell-femtocell network.

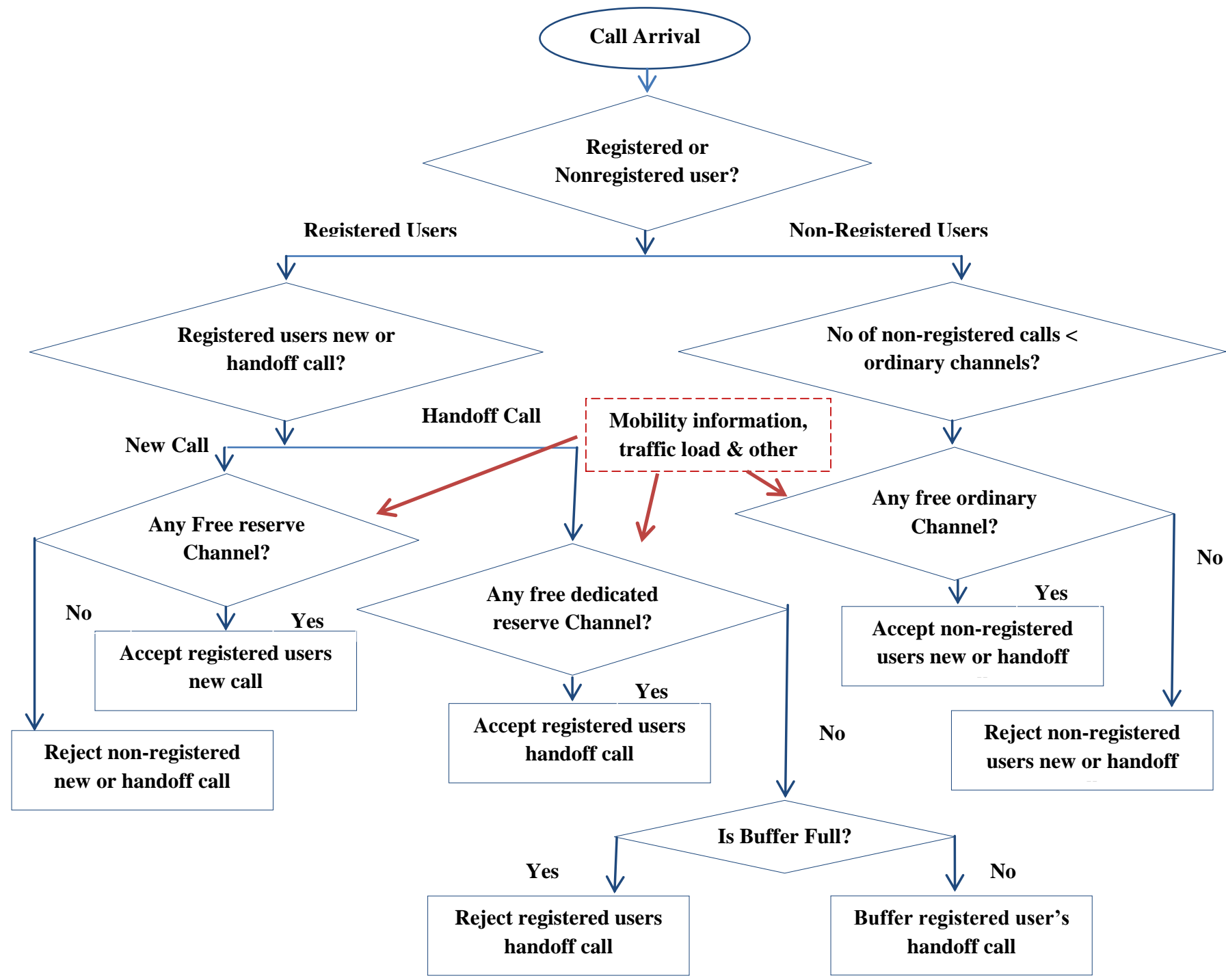

Fig 2: Handover Prioritized Call Admission Control for integrated macrocell-femtocell network

\section{SYSTEM MODEL}

For this work, we have considered integrated macrocellfemtocell network which works on WCDMA technology and femtocells working in hybrid access mode [9]. In section 2, we have already seen Markov model which calculates blocking probability. We will use 4 dimensional markov chains to address three different types of calls for registered \& non-registered users. First type is registered users handoff call, second registered user's new call \& finally non- registered users new as well as handoff call. These types even talk about channel priority which we decided to achieve better customer satisfaction. Consider cell has $\mathrm{N}$ number of total channels \& Buffer Size is B channels. New call arrival rate and handoff calls arrival rate for registered users are Poisson distributed, and it has arrival rates $\lambda \mathrm{r}$ and $\mathrm{hr}$ respectively. Thus, the total call arrival rates for registered users are $\lambda \operatorname{tr}=\lambda \mathrm{r}$ $+\mathrm{hr}$. Non-registered users new as well as handoff calls arrival rate also Poisson distributed with arrival rates $\lambda$ a. Hence, total call arrival rate of registered \& non-registered calls in a cell 
are $\lambda \mathrm{t}=\lambda \operatorname{tr}+\lambda \mathrm{a}$. Total $\mathrm{N}$ channels are divided into 3 parts: $(\mathrm{N} 1<\mathrm{N} 2<\mathrm{N}) \&$ additional Buffer B.

The balance equation of steady-state [P (st)] for Markov chain model is given by

$$
\mathrm{P}(\mathrm{st}) \begin{cases}\frac{\mathrm{P}^{\mathrm{i}}}{\mathrm{i} !} P 0 & \text { If } \mathrm{i} \leq \mathrm{N} 1 \\ (\alpha)^{-\mathrm{N} 1} \frac{(\mathrm{P} \alpha)^{i}}{\mathrm{i} !} P 0 & \text { if } \mathrm{N} 1<\mathrm{i} \leq \mathrm{N} 2 \\ (\alpha)^{-\mathrm{N} 1}(\alpha / \alpha 2)^{N 2} \frac{(\mathrm{P} \alpha 2)^{i}}{\mathrm{i} !} P 0 & \text { If } \mathrm{N} 2<\mathrm{i} \leq \mathrm{N}\end{cases}
$$

Where,

$$
\begin{aligned}
& \mathrm{P}=\lambda \mathrm{t} / \mu \\
& \alpha=\lambda \operatorname{tr} / \lambda \mathrm{t} \\
& \alpha 2=\mathrm{hr} / \lambda \mathrm{t}
\end{aligned}
$$$$
\mathrm{i}=\text { current state or channel }
$$$$
\mathrm{P} 0=\text { Probability that all channels are free }
$$

$$
\begin{aligned}
& \mathrm{P} 0=1 /\left[\sum_{i=0}^{N 1-1}\left(\frac{(\mathrm{p})^{\mathrm{i}}}{\mathrm{i} !}\right)+(\alpha)^{-\mathrm{N} 1} \sum_{i=N 1}^{N 2-1}\left(\frac{(\mathrm{p} \alpha)^{\mathrm{i}}}{\mathrm{i} !}\right)\right. \\
& \left.+(\alpha)^{-\mathrm{N} 1}\left(\frac{\alpha}{\alpha 2}\right)^{\mathrm{N} 2} \sum_{i=N 2}^{N}\left(\frac{(\mathrm{p} \alpha 2)^{i}}{\mathrm{i} !}\right)\right]^{2}
\end{aligned}
$$

With this 4 dimensional Markov chain model, we can calculate blocking probability for handoff $\&$ new calls for registered \& Non-registered users as below.

1) Blocking Probability of handoff calls for registered user

$$
\mathrm{Bhr}=(\alpha)^{-\mathrm{N} 1}\left(\frac{\alpha}{\alpha 2}\right)^{\mathrm{N} 2}\left(\frac{(\mathrm{p} \alpha 2)^{\mathrm{N}}}{\mathrm{N} !}\right) * \mathrm{P} 0
$$

2) Blocking Probability of new calls for registered user

$$
\mathrm{Bnr}=(\alpha)^{-\mathrm{N} 1}\left(\frac{\alpha}{\alpha 2}\right)^{\mathrm{N} 2} \sum_{i=N 2}^{N}\left(\frac{(\mathrm{p} \alpha 2)^{\mathrm{i}}}{\mathrm{i} !}\right) * \mathrm{P} 0
$$

3) Blocking Probability of new and handoff calls for nonregistered user

$\mathrm{Bhn}=\sum_{i=N 1}^{N 2-1}\left(\frac{(\mathrm{p} \alpha 2)^{i}}{\mathrm{i} !}\right) * \mathrm{P} 0+$

$(\alpha)^{-\mathrm{N} 1}\left(\frac{\alpha}{\alpha 2}\right)^{\mathrm{N} 2} \sum_{i=N 2}^{N}\left(\frac{(\mathrm{p} \alpha 2)^{\mathrm{i}}}{\mathrm{i} !}\right) * \mathrm{P} 0$

In addition to this, Buffer channels are added to safeguard handoff calls of registered user. Blocking probability of handoff calls for registered user with buffer is further reduced \& calculated by

$\mathrm{Bhrb}=(\alpha)^{-\mathrm{N} 1}\left(\frac{\alpha}{\alpha 2}\right)^{\mathrm{N} 2}\left(\frac{(\mathrm{p} \alpha 2)^{(\mathrm{N})}}{(\mathrm{N}+\mathrm{B}) !}\right) * \mathrm{P} 0$

Let's see simulation model for proposed Call admission control policy in next subsection.

\subsection{Simulation Model}

The Proposed algorithm is simulated with MATLAB. Below fig. 3 highlights the simulation framework for this approach. We will consider network scenario where multiple femtocells are deployed over coverage area of macrocell. Measurement report of MS, access mode of femtocell, registered users list, Call request \& earlier traffic load along with mobility/other related information are inputs for $\mathrm{HO}$ decision as well call admission control. We will get blocking probability for new \& handoff calls. As highest priority is given to registered users handoff calls we can see that its blocking probability is much lesser than new call \& non-registered user calls. It will further reduce with buffer.

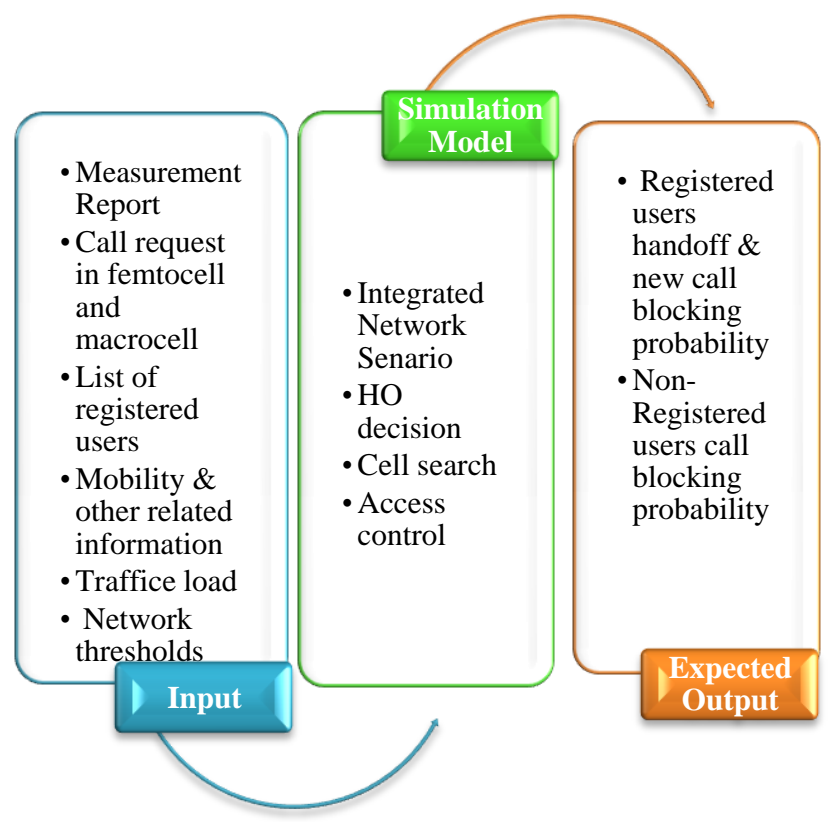

Fig 3: Simulation Framework

Let's see simulation parameters used for this approach in below table 1 .

Table 1. Simulation Model

\begin{tabular}{|l|l|}
\hline Network Scenario & $\begin{array}{l}\text { 2 macrocell with Multiple } \\
\text { Femtocell (Implemented using } \\
\text { x-y Coordinate system) }\end{array}$ \\
\hline $\begin{array}{l}\text { Radius of macrocell } \\
\text { coverage area }\end{array}$ & Rm \\
\hline $\begin{array}{l}\text { Radius of femtocell } \\
\text { coverage area }\end{array}$ & Rf \\
\hline $\begin{array}{l}\text { distance from macrocell to } \\
\text { in meters }\end{array}$ & dm (Random) \\
\hline $\begin{array}{l}\text { distance from macrocell to } \\
\text { MS meters }\end{array}$ & df (Random) \\
\hline hysteresis in dBm & $0 / 3$ \\
\hline Total Capacity & Ctotal \\
\hline Used Capacity & Cused (Random) \\
\hline $\begin{array}{l}\text { Available capacity of } \\
\text { femtocell }\end{array}$ & Cf= Ctotal-Cused \\
\hline $\begin{array}{l}\text { Available capacity of } \\
\text { macrocell }\end{array}$ & Cm= Ctotal-Cused \\
\hline $\begin{array}{l}\text { Distance between } \\
\text { macrocell \& femtocell }\end{array}$ & Poisson Distribution \\
\hline Call arrival rate & \\
\hline
\end{tabular}




\begin{tabular}{|l|l|}
\hline User Mobility & Random waypoint \\
\hline Bandwidth & $10 \mathrm{MHz}$ \\
\hline
\end{tabular}

\section{SIMULATION RESULT \& ANALYSIS}

For simulation we have created one network scenario of 2 macrocells (MBS) \& multiple femtocells (FBS) deployed in coverage area of macrocell. Blue circle shows coverage area of macrocell \& femtocell. The proposed approach is applied on this network which works on WCDMA technology.

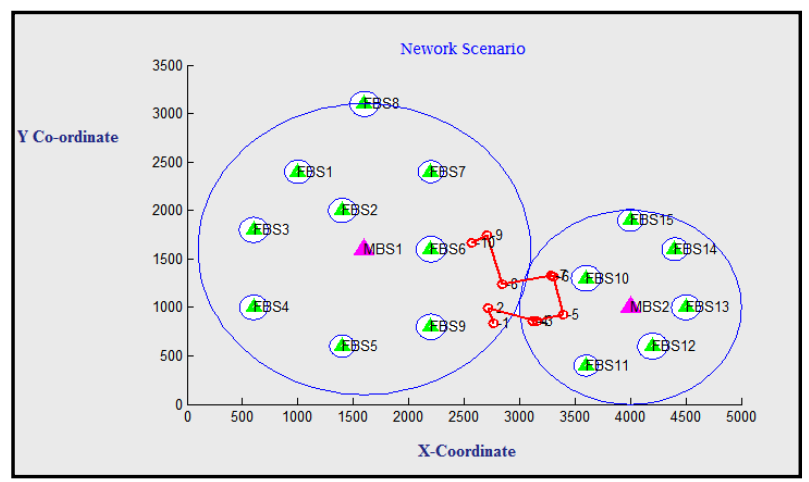

Fig 4: Network Scenario

In above network scenario, user mobility is shown by red line graph. Random waypoint model is used to define user mobility.

Proposed policy gives maximum priority to handoff calls of femtocell registered user to reduce handoff blocking probability. It is achieved by reserving channels for handoff \& buffer mechanism. Simulation result of proposed policy shown by Fig. 5 .

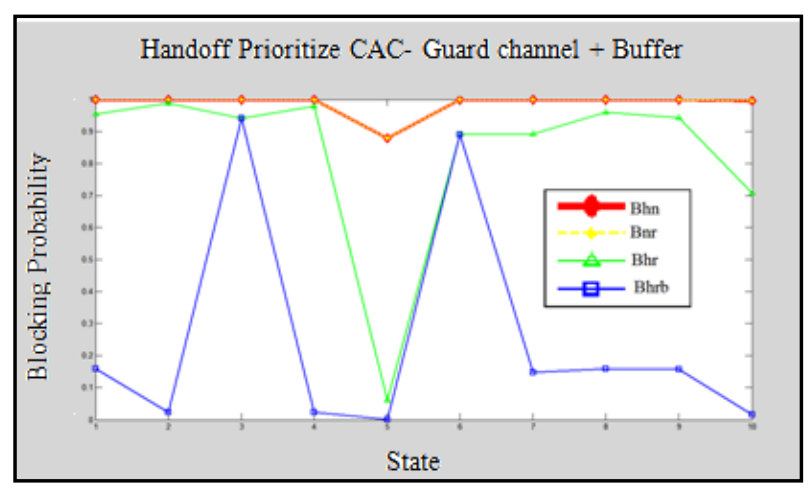

Fig 5: Proposed CAC Policy Performance Result

In above graph, overlapping red \& yellow lines are showing blocking probability of non-registered users call \& registered users new call simultaneously. Registered users handoff call blocking probability with early blocking or guard channel policy is shown by green line \& finally blue line shows registered users handoff call blocking probability, result of hybrid CAC approach which combines Guard channel policy and buffer or queuing. From Above simulation result we can clearly see that proposed call admission control reduces handoff call blocking probability over others by reserving channels. Fig 6 highlights comparison of proposed approach with non-prioritize call admission control.

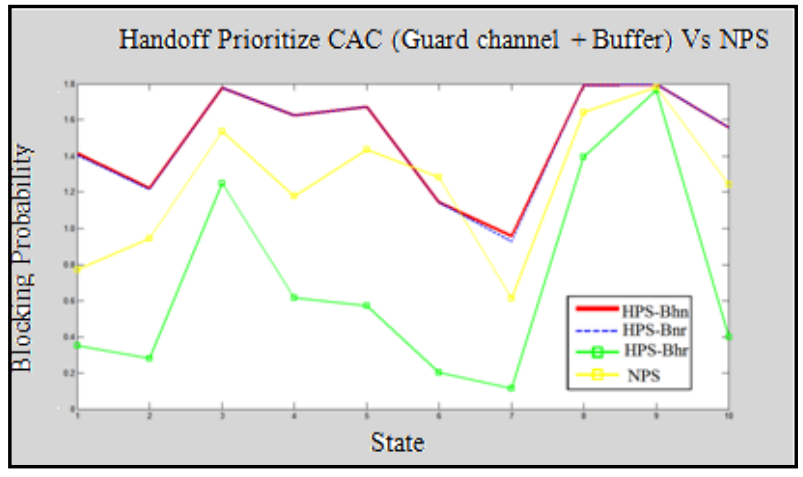

Fig 6: Proposed CAC Policy V/S NPS

Yellow line shows blocking probability of new as well as handoff call with non-prioritize call admission scheme (NPS). In NPS, all channels are shared by all users \& for all wireless transaction so blocking probability is same. Green line is handover blocking probability with proposed approach which is much lesser than NPS but we can see that new calls blocking probability shown by red line is increased with proposed policy as we have less channels for them now.

Proposed policy divides total channels into 3 subsets \& reserves few channels only for handoff. The threshold for this subset or number of channels reserved for handoff can be decided by service provider based on requirement; hence the proposed approach is very useful for better resource utilization too. If we change ratio of reserved channels over total channels then handoff \& new call blocking probability for registered \& non-registered users' changes. The study of this relation will help service provider for capacity forecast.

\section{CONCLUSION}

In this paper, we investigate Handoff prioritize call admission control strategy for integrated macrocell-femtocell network. The simulation has revealed that:

1. This approach helps us to reduce handoff blocking probability for registered users over new call \& nonregistered users call.

2. The Handoff blocking probability further reduces with buffer

3. Blocking Probability of Handover calls of proposed algo is much lesser than handover blocking probability of non-priorities scheme but registered users new \& nonregistered users call blocking probability of proposed scheme is higher.

4. We can achieve better resource utilization \& customer satisfaction with Optimal Number of Guard channels. Proposed algorithm helps us to find optimal number of guard channels.

The proposed handoff prioritization CAC policy, regardless of the numbers of channels reserved for handoff is a control limit policy or threshold policy to reduce handoff drop and to improve customer satisfaction. But the resource utilization gets impacted by the numbers of reserved channels for handoff. Hence proposed algorithm proves itself very useful for Optimal Number of Guard channels, capacity forecast \& management.

As proposed policy gives higher priority to handoff calls, blocking probability of new call increasing. If we don't have handoff calls request, reserved channels for hand off may get wasted. Instead of it we can work on accepting new calls with 
certain probability $\beta$ if they cross threshold set for new calls as a future scope. This way it will reduce new call blocking probability without affecting handoff call drop as well as blocking probability.

\section{REFERENCES}

[1] Cisco, "Cisco Visual Networking Index: Global Mobile Data Traffic Forecast Update, 2016-2021”, White paper, Feb 2017

[2] Jeffrey G. Andrews, Holger Claussen, Mischa Dohler, Sundeep Rangan, Mark C. Reed, "Femtocells: Past, Present, and Future" IEEE Journal on Selected Areas in communications, Vol-30, No-3, April 2012.

[3] S. Padmapriya , M. Tamilarasi, "A case study on femtocell access modes", Elsevier, 2016

[4] Majid Ghaderi and Raouf Boutaba, "Call Admission Control in Mobile Cellular Networks: A Comprehensive Survey", University of Waterloo

[5] Wenlong Ni, "Optimal Call Admission Control Policies in Wireless Cellular Networks Using Semi Markov Decision Process", December 2008.

[6] 10. A. Halim Zaim, Istanbul University, "A Markov Model to Calculate New and Hand-off Call Blocking Probabilities in LEO Satellite Networks", Journal of Research and Practice in Information Technology, Vol. 35, No. 4, November 2003.

[7] Hamid Beigy, M. R. Meybodi, "A Two-threshold guard channel scheme for minimizing blocking probability in communication networks", Received: March 4, 2003 Accepted in Revised Form: June 10, 2004

[8] Oyebisi T.O, Ojesanmi O.A, "Development of congestion control scheme for wireless mobile network", Journal of Theoretical and Applied Information Technology, (C) 2005 - 2008 JATIT.
[9] Woojune Kim, "Femtocell Network Architecture", Whitepaper, Airvana, May 2010

[10] Joseph Boccuzzi, Michael Ruggiero, "Femtocells: Design \& Application", McGraw-Hill Companies., 2011

[11] Jie Zhang, Guillaume da la Roche, “ Femtocell Technologies and Deployment”, John Wiley \& Sons Ltd, 2010

[12] Mostafa Zaman Chowdhury, Yeong Min Jang, ZJ Haas, "Network evolution and QoS provisioning for integrated femtocell/macrocell networks". Int. J. Wirel. Mobile Netw. 2(3), 1=16(2010)

[13] Jaime Ferragut, Josep Mangues-Bafalluy, Jose Nunez=Martinez \& Frank Zdarsky, "Traffic and Mobility Management in Networks of Femtocells", Springer Science+Business Media, LLC 2012. Published online: 11 August 2012

[14] Tijane Fatima Zohra, Badri Saadane Rachid, Mohammed Wahbi and Mbarki Samir, "Call Admission Control Scheme and Handover Management in LTE FemtocellMacrocell Integrated Networks", Computer and Information Science; Vol. 8, No. 1; 2015

[15] Oyebisi T.O, Ojesanmi O.A, "Development of congestion control scheme for wireless mobile network", Journal of Theoretical and Applied Information Technology, () 2005 - 2008 JATIT.

[16] Hamid Beigy, M. R. Meybodi, "A Two-threshold guard channel scheme for minimizing blocking probability in communication networks", Received: March 4, 2003 Accepted in Revised Form: June 10, 2004

[17] Georgios I. Tsoropoulos, Dimitrios G. Stratogiannis and Eirini Eleni Tsoropoulou, "Call Admission Control in Mobile and Wireless Network" National Technical University of Athens Greece, Published on 2010-01-01 